\title{
Research on the Current Situation, Problems and Development Trend of Software Reuse Technology
}

\author{
Shi Lei \\ Teaching and research section of Information management, \\ Information and Technology College \\ Jilin Agricultural University \\ Changchun, China \\ shil0212@sina.com
}

\author{
$\mathrm{Su} \mathrm{Yi}$ \\ Fuxin animal disease prevention and control center \\ Fuxin, China \\ 419998007@qq.com
}

\begin{abstract}
Software reuse is an important technology in the world since 1980s. It is considered as a technology that can increase software productivity and provide important support for software development industrialization. Software reuse is involved in a very broad field of technology research, the research results can be applied in business, medical and military etc., it is one of the most important research hotspot in software engineering. This paper introduces the history of the development of software reuse, the basic concept and application status, analyzes the existing problems of computer software reuse technology and proposes solutions, infers the future development trend of the technology of software reuse.
\end{abstract}

Keywords—Software; reuse; software reuse; software crisis

\section{INTRODUCTION}

At initial stage, the software development cycle is long, the reliability is bad, and the production cost is high, it is difficult to maintain, the system cannot completely meet the needs of users, in order to solve the software crisis, improve the production efficiency of software development, we put forward the concept of software reuse [1].

Twenty-first Centuries is the era of digital information, computer software has become an essential part of people's lives. Software, as the carrier of the network world, makes the Internet bring a colorful and magical world for people's life and become an integral part of today's society [2-3]. However, the traditional software system development is a rather lengthy and tedious process. It takes a lot of time and effort to test and maintain software even if successful development, how to develop and test new computer software easily and quickly is the problem that all software engineers need to solve in the process of software development. Software reuse is a good solution to this problem. Since the origin of software reuse technology, it has gradually become an important research focus in the field of software development and application [4$5]$.

This paper first introduces the current development of software reuse technology, and then introduces the problems and reasons of software reuse technology in the process of using, finally introduces how to solve the problems of software reuse and the development trend of the technology.

\section{RESEARCH BACKGROUND}

In October 1968, in response to the first software crisis, the Technology Committee of the North Atlantic Treaty Organization (NATO) funded a software engineering conference. The term "software engineering" was formally defined and "software engineering" emerged as a new discipline to study and overcome the software crisis [6-7]. In 1979, Lanergan published a paper summarizing the research of a software reuse project, which made the research of software reuse get people's attention again.

In 1983, sponsored by ITT, Ted Biggerstaff and Alan Perlis Newport in the United States organized the first symposium on software reuse, then in 1984 and 1987, the international authoritative journal IEEE Transactions on Software Computer Engineering and IEEE Software were published about software reuse of the album, the first session of the 1991 International Conference on Software Reuse (IWSR) held in Dortmund in Germany, the second session of IWSR held in Italy Lucca in 1993, from the beginning of 1994 third, renamed the International Conference on software reuse [8-9].

In the mid-80s, people realized that reuse was one of the key factors in software design, and there were 3 trends in the practice of software reuse, first, the practice of reusing software reuse in software industry should not only consider the factors of technology, but also consider the factors of management. Two is to integrate the reuse technology into the software development process, and to study the formalization of software process. Three is to standardize domain analysis and develop methods and tools to support domain analysis.

Since the software reuse technology was put forward, it has gone through three stages of development, including structured programming, object-oriented technology and software construction technology [10]. The object-oriented technology has been deep in development of application software, because it can build through the software model, the software component of the abstract space design, reasonable assembly for software components with different functions, the reorganization of the software features more complete, reusable software element more software the development of more help to improve the quality of software. Software reuse technology can greatly enhance the productivity of software 
development, improve the software reliability, reduce the cost of software maintenance, and universities and research institutions, software enterprises have paid attention to the research and experiment of software reuse.

\section{Current Situation AND PROBlems Analysis}

\section{A. Software Reuse Technology}

Software reuse is the process of reusing identical or similar software components during two or more different software development processes. Simply put, it is the use of existing software components to form new software systems. Broadly speaking, software reuse can be divided into the following three levels: knowledge reuse, methods and standards reuse, software component reuse.

1) Software Reuse Process

The software reuse process chart is as follow Fig. 1.

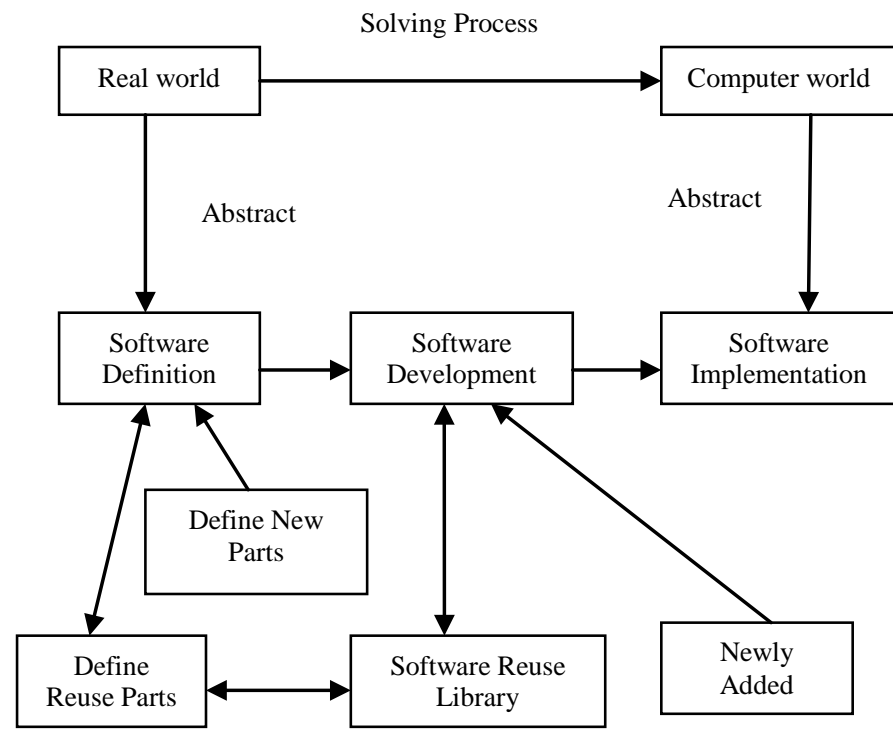

Fig.1. Software reuse process chart

\section{2) Reusable Components}

The reusable components of software reuse include the following:

Data, using a standard data interchange format, facilitates software reuse, especially in the current stage where sound and images are also defined as data. When data is in standard form, new applications can use data without formatting and content in advance.

Program structure, effective reuse requires a structural starting point, not just a modular combination of connections.

Programming, which refers to the construction methods that can be reused to describe common application design types and reference materials for general programming? This reuse helps to migrate an application system to a completely different hardware and software platform.

Program analysis results and the analysis model of software systems can also be reused. This kind of reuse is applicable to software development where the demand has not changed, but the system structure has changed.

\section{3) Features}

The independence of each component is strong. The function of the module must be complete and unique, and after many accurate tests, it is recognized as correct. It is an independent package that is free from or affected by outside interference.

The structure of software is clear. The software component should have a reasonable structure level, being easy to understand and easy to read, and the interface must be simple, clear and reliable.

High plasticity of component. The reuse part needs to be able to adapt to various operating environments, and provides convenience for adding or modifying the existing component usage mechanism for adapting to specific requirements.

\section{4) Types of Technology}

Software composition technology a technique that combines reusable software components together to form new reusable components or software systems according to certain rules. The technology is characterized by reusable software components that will be used as passive component modules and remained the same throughout the integration process.

Software generation technology, based on a certain generation mechanism and formalized software function description, generates the object program automatically by the generator system. The reusable component is used by this method is the code board of the generator itself or hidden in the template of the transform rule set. This technology has a high level of abstraction and requires the support of reusable software libraries and knowledge bases.

Object oriented software technology can improve the reusability of software by improving the aggregation of methods, reduce the scale and couple degree of methods, and use inheritance mechanism, delegation and encapsulation. Object oriented software technology provides the basic technical support for the development of reuse technology. Using object-oriented technology, software reuse can be realized more conveniently and effectively.

\section{B. Existing Problems and Analysis}

The software reuse technology has been accepted, but early can only be applied to some small software development process, has not been widely used, especially in the face of large and complex software development process. There are many factors that affect the development of software reuse technology, which can be briefly summarized as follows:

1) The construction of software reuse components. In today's software development environment where multiple development languages and development methods exist, it is a problem to identify software components, using language and methods. The most method and technology of the assumption that in certain areas of memory in one or several experts on the field, they can effectively capture and reuse components in the field of construction. However, in the process of information accelerating and software applications emerging, evolving and 
merging, this assumption is too optimistic and is not established in most practical situations.

2) The understanding and modification of software reuse components. The software reuse component is to understand how to understand existing software components, confirm the development method of software component, the original internal structure, function, style and other related characteristics. This process requires very high software identification capability and considerable workload. How to properly apply and modify the software reuse part according to the characteristic requirements of the new software development, how to adapt to the new software environment, is a difficult problem in the technical field.

3) The cost of building reusable libraries. Before the reuse of software, it is necessary to establish a perfect reusable library, and the manpower and material resources which are needed to build the database are the additional investment that the general development institution is unwilling to do. Each project development team has determined the project budget and need to complete the goal, all need to construct reusable components not all software development, the high investment makes the project development institutions in general to construct, which has become a big problem to establish the reusable library.

4) The problem of software developers. The use of software reuse technology is complicated, usually software developers prefer independent RE development, RE start operations or calculation, is not willing to rely on existing resources for operation and operation procedures, the basic object of language, machine, environment details cannot achieve smooth connection, will add great difficulty to the development process.

5) Software copyright issues. From the point of view of software development, the establishment of a large-scale software component base will need a large number of software copyright material, which is automatic protection, no registration, no relevant information that are available to the public, which makes software developers hard to know the development of software. In this way, when software developers build software libraries, it is inevitable to dissect and analyze other people's software works. In this process, software developers must copy some of the program works, but from the perspective of copyright protection, the copy should be forbidden, all procedures can work according to the copyright law against this copy, thus forcing the software developers and other software completely develops different software to increase the cost of software development. These factors are a great obstacle to the development of software reuse.

\section{SOlutions AND TheIR DEVELOPMENT PROSPECTS}

According to statistics, in the development of a new application system, $40 \% \sim 60 \%$ of the code is to repeat the previous similar system components, repeated proportion, sometimes even higher. Therefore, software reuse can save the cost of software development and improve the efficiency of software production. The contrast table of using and without using software reuse in development software is Tab.1.
TABLE I. USING AND WITHOUT USING SOFTWARE REUSE

\begin{tabular}{ccc}
\hline & without software reuse & Software reuse \\
\hline $\begin{array}{c}\text { Development } \\
\text { efficiency } \\
\text { Development } \\
\text { cycle }\end{array}$ & low & high \\
Cost input & long & Short \\
Required & many & less \\
personnel & many & less \\
Problems in & Probably more & May less \\
operation & & \\
\hline
\end{tabular}

\section{A. The Solutions}

In order to solve the existing problems of software reuse technology, two aspects of technology and personnel need to be considered. First, in terms of technology, we should make further improvement and development of all kinds of methods of software reuse and the existing technology, and avoid blind expansion of feature model, should be clear around to enhance the effective organization of the field of reusable components and management of such a core objective to explore a more direct and easy to use modeling mechanism of formation, component library system, which is similar to the database management system, Reuse component library information cannot be classified too complex, it should help in the shortest time to retrieve a only minimal changes of reusable software components. It should have the basic features and functions of the database, and at the same time, a system standard for reusing component representation, retrieval and storage is needed. In order to make the system support reuse enough to meet the requirements of the times change rapidly, we should fully understand the importance of reusable domain, analysis of the domain of software reuse, It can lay a good foundation for object oriented software design based on class libraries.

Secondly, in the human factor, we must break the existing methods and technical implementation of the dependence on a few experts. Because the software became more widespread, the size and diversity of a software product of stakeholder groups is also increasing continuously, a few experts have been very difficult to cope with software reuse in many areas to implement the task, the possible solution is to adopt the way of group collaboration on the feature model and its relationship between tracking and implementation of products modeling and evolution. In theory, some experts in the collaborative support, as long as the participants in the group level with sufficient domain knowledge, and to continue to participate in the modeling activities, can make the modeling results in high quality and continuous evolution, In reality, the development of Internet and Web technology for large-scale human group collaboration provides basic technical support, and various groups of intelligent applications based on Web demonstrate the feasibility of this solution, the key tasks and activities to construct feature oriented software reuse in the system become open, incremental and parallel that will no longer be an impossible development environment. In terms of copyright, all computer and law experts should get together to explore, governments introduced a special solution for the establishment and use of software reuse library copyright problems involved in the laws and regulations, so as to improve the current situation of the development for the maintenance of the interests of developers and obstacles of software reuse technology. 


\section{B. The Development Prospects}

At present, more and more software systems in our country are developed by software reuse technology, which revolves around the production, management and assembly of software, a considerable scale of software reuse development template tool market will be formed. With the extension and deepening of software reuse, reuse theory has penetrated into various industries, including some special fields, such as military field, aviation field and so on. Because of the particularity of the field, the existing mature theory and technology cannot fully meet the requirement of these areas, so the establishment of software reuse technology and template for the special field based on the mature theory and technology is the inevitable trend. The use of software reuse components in software development is the future direction of software development. It is an effective way to improve the efficiency and quality of software development, and is a social software development method. The continuous development of software reuse technology will make the industrialization of software development, design and production possible. With the continuous improvement of technology, it will lead to profound changes in the field of software engineering. Especially in recent years, the rise of the network distributed development for large software brings the software product richly endowed by nature conditions. The internationalization of software products has also been improved .the software reuse technology will have broad prospects for development of good.

\section{Conclusions}

Software reuse is like standing on the shoulders of a previous generation", Software reuse can greatly improve software productivity, reduce development costs and ensure the quality of software. It is an effective way to solve software crisis, Software reuse technology is the main technical support of rapid development software, and it is also the basic premise to support software engineering to realize industrialization.
Developing software by reusing technology greatly reduces software costs and many repetitive tasks, improves software productivity and shortens development cycles. In the future work of software development we should promote the objectoriented technology, the application of artificial intelligence technology, Internet technology in the research of the software reuse. In addition, the development of automated tools and software support environments for reuse of generated software should also be promoted. It is believed that in the near future, software reuse technology will become an influential technology in the IT industry.

\section{REFERENCES}

[1] Yupu Song, Liu Min. "To Explore Several Key Issues in Software Reuse Technology”. Value Engineering, 2011(30), pp. 151-152.

[2] Gao Hao. "Research on software reuse and software component technology”. Electronic Technology \& Software Engineering, 2015(10), pp. 102-102.

[3] Caiping Sun. "Research on software reuse technology based on software development”. Education Teaching Forum, 2012 (39), pp. 247-249.

[4] Xie Fang. "Research on component based software reuse technology". Computer CD Software and Application, 2013(10), pp. 81-82.

[5] Shi Yan. "Application of reuse technology in software engineering”. China Science and Technology Information, 2010(3), pp. 113-114.

[6] Yanke Dong. "The research of computer software reuses technology". Digitization User, 2013(4), pp. 48-50.

[7] Wenjuan Hu. "On how to improve the reusability of JAVA code". China New Telecommunications, 2017(2), pp. 102-102.

[8] Zhang Wei, Mei Hong. "Feature oriented software reuse technologyDevelopment and present situation”. Chinese Science Bulletin, 2014(1), pp. 21-42.

[9] Dandan Wang, Chen Kang. "Current situation and development trend of software engineering technology”. China Computer \& Communication, 2016(6), pp. 50-51.

[10] Li Zhen. "Research on the development trend of software engineering technology”. Telecom World, 2017(3), pp. 230-231. 